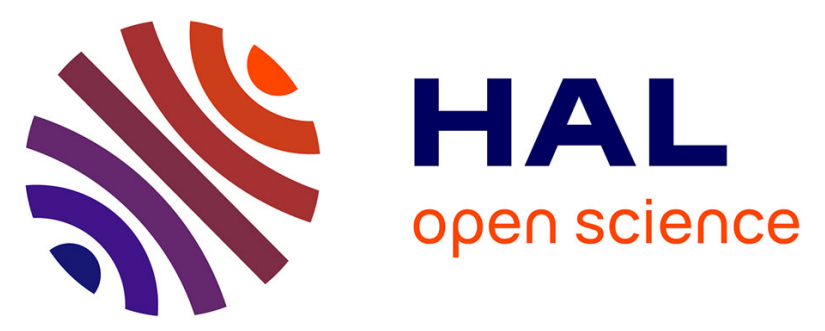

\title{
Cooperative Effects Dominating the Thermodynamics and Kinetics of Surfactant Adsorption in Porous Media: From Lateral Interactions to Surface Aggregation
}

Zaineb Zaafouri, Daniela Bauer, Guillaume Batôt, Carlos Nieto-Draghi, Benoit Coasne

\section{To cite this version:}

Zaineb Zaafouri, Daniela Bauer, Guillaume Batôt, Carlos Nieto-Draghi, Benoit Coasne. Cooperative Effects Dominating the Thermodynamics and Kinetics of Surfactant Adsorption in Porous Media: From Lateral Interactions to Surface Aggregation. Journal of Physical Chemistry B, 2020, 124 (47), pp.10841-10849. 10.1021/acs.jpcb.0c08226 . hal-03118492

\section{HAL Id: hal-03118492 \\ https://hal-ifp.archives-ouvertes.fr/hal-03118492}

Submitted on 22 Jan 2021

HAL is a multi-disciplinary open access archive for the deposit and dissemination of scientific research documents, whether they are published or not. The documents may come from teaching and research institutions in France or abroad, or from public or private research centers.
L'archive ouverte pluridisciplinaire HAL, est destinée au dépôt et à la diffusion de documents scientifiques de niveau recherche, publiés ou non, émanant des établissements d'enseignement et de recherche français ou étrangers, des laboratoires publics ou privés. 


\title{
1. Cooperative Effects Dominating the Thermodynamics and Kinetics of Surfactant Adsorption in Porous Media: From Lateral Interactions to Surface Aggregation
}

\author{
${ }_{4}$ Zaineb Zaafouri, Daniela Bauer,* Guillaume Batôt, Carlos Nieto-Draghi, and Benoit Coasne*
}

5 ABSTRACT: Surfactant adsorption in porous media remains poorly 6 understood, as the microscopic collective behavior of these amphiphilic 7 molecules leads to nonconventional phenomena with complex underlying 8 kinetics/structural organization. Here, we develop a simple thermodynamic 9 model, which captures this rich behavior by including cooperative effects to 10 account for lateral interactions between adsorbed molecules and the

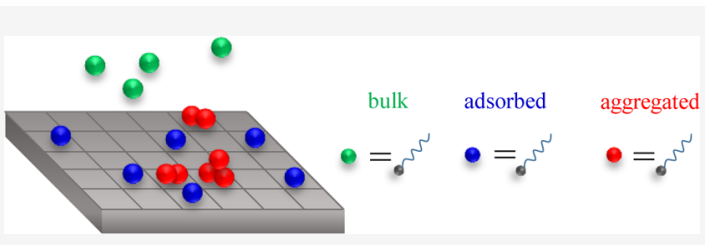
11 formation of ordered or disordered self-assemblies. In more detail, this model relies on a kinetic approach, involving adsorption/ 12 desorption rates that depend on the surfactant surface concentration to account for facilitated or hindered adsorption at different 13 adsorption stages. Using different surfactants/porous solids, adsorption on both strongly and weakly adsorbing surfaces is found to 14 be accurately described with parameters that are readily estimated from available adsorption experiments. The validity of our physical 15 approach is confirmed by showing that the inferred adsorption/desorption rates obey the quasi-chemical approximation for lateral 16 adsorbate interactions. Such cooperative effects are shown to lead to adsorption kinetics that drastically depart from conventional 17 frameworks (e.g., Henry, Langmuir, and Sips models).

\section{INTRODUCTION}

18 Surfactants, which are amphiphilic molecules combining a 19 hydrophilic head with a hydrophobic tail, constitute an 20 important class in Soft Matter. Besides their ability to decrease 21 surface tension by getting adsorbed at interfaces, an important 22 property of surfactants in water is to exhibit a complex phase 23 diagram including a miscibility gap (a temperature/concen24 tration range where demixing occurs from water). ${ }^{1}$ Even in the 25 miscibility region, there exists a critical micelle concentration $26(\mathrm{cmc})$ above which surfactants form ordered mesoscopic 27 assemblies-typically spherical micelles where the surfactant 28 tails are inside the micelle, while polar or charged heads are at 29 the micelle external surface in contact with water (Figure 1). 30 Owing to their tendency to form micelles and reduce surface 31 tension, surfactants are at the heart of many applications where 32 they are used as detergents, dispersants, emulsifiers, and so 33 forth. $^{2-4}$ In particular, in heterogeneous solutions such as oil/ 34 water systems, they are used to trap oil droplets inside the core 35 of surfactant micelles. In many situations, such a phase 36 separation occurs in porous media or in the vicinity of solid 37 surfaces, an important example being the use of surfactants to 38 untrap important oil amounts located in oil reservoirs/ 39 rocks. $^{5-7}$

40 The rich thermodynamic behavior of surfactants results from 41 competing molecular interactions between the different42 hydrophobic and hydrophilic-groups, which combine with 43 large entropy effects for such molecules. ${ }^{8}$ However, despite 44 such an intrinsic complexity, the phase behavior of bulk 45 surfactants is reasonably well understood with available formalisms to describe phenomena such as self-assembly and 46 phase separation/transition and also nonintuitive temperature 47 effects on liquid immiscibility, solubility, and micellization. ${ }^{9-11} 48$ In contrast, the physical behavior of surfactants confined in 49 porous materials or close to solid surfaces still challenges 50 existing frameworks. ${ }^{12}$ Adding free energy contributions 51 resulting from the head/surface and tail/surface intermolecular 52 interactions leads to intriguing effects such as inverse 53 temperature adsorption and also surface transitions between 54 disordered and/or ordered mesoscopic assemblies (e.g., 55 bilayers, hemi-micelles, vesicles, and elongated micelles). ${ }^{9,13-15} 56$ The situation is even more puzzling as the observed adsorption 57 type depends specifically on the solid chemistry (e.g., surface 58 affinity/groups with possible amphoteric charges), surfactant 59 molecules (e.g., apolar/polar, cationic/anionic), and thermo- 60 dynamic/solution conditions (e.g., concentration, temperature, 61 and presence of an electrolyte). ${ }^{16-19}$

As a result of this complexity, most of the experimental 63 literature on surfactant adsorption focuses on a given family of 64 surfaces or surfactants. In particular, significant research effort 65 has been devoted to unraveling the structural mechanisms 66 followed upon adsorption at increasing concentrations. ${ }^{20-24}$ By 67 


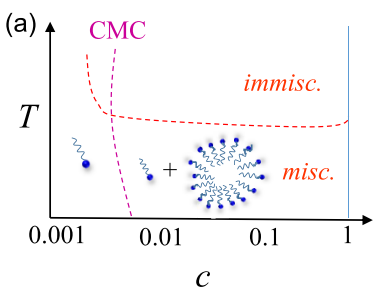

(b)

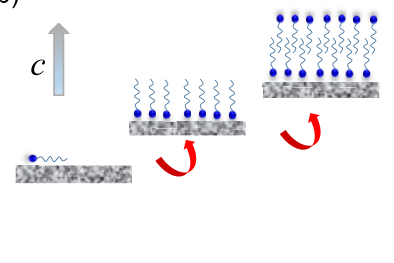

(c)

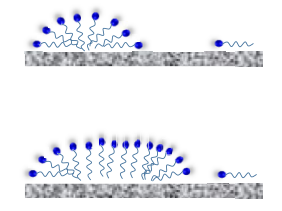

Figure 1. (a) Temperature-concentration $(T, c)$ phase diagram of bulk surfactant solutions. The surfactant monomer is pictured as a hydrophilic head (blue sphere) combined with a hydrophobic chain (grey segment). A miscibility gap separates the high/low $T$ regions with solvent/surfactant miscibility only observed at low $T$ and $c$. In the miscibility range, for $c$ smaller than the so-called $\mathrm{cmc}$, the monomers are solubilized in the solvent phase. For $c>c m c$, the monomers coexist with micelles. $(b, c)$ Orientation of surfactant molecules and possible surface aggregates obtained at a solid surface. (b) corresponds to the stepped Langmuir adsorption isotherm with the formation of an adsorbed monomer layer followed by film reorientation and growth. (c) illustrates other ordered or disordered self-assemblies coexisting with isolated adsorbed monomers.

68 combining thermodynamic measurements with structural 69 analysis, some authors proposed advanced scenarios to 70 rationalize step adsorption and/or S-shaped adsorption 71 isotherms observed experimentally. ${ }^{25,26}$ As illustrated in Figure $72 \mathrm{lb}$, such mechanisms involve the adsorption of isolated 73 monomers followed by the formation of a monolayer, which 74 eventually transforms into more complex structures (e.g., 75 bilayer, hemimicelle, and vesicle) upon increasing the 76 surfactant concentration. ${ }^{27}$ However, although such combined 77 structural/thermodynamical studies provide a robust descrip78 tion for a broad class of solid/surfactant situations, there is a 79 number of systems that lead to more complex data departing 80 from such a generic picture. $^{28,29}$ In particular, data for 81 surfactants in various silica-based porous materials display 82 complex adsorption mechanisms and kinetics involving the 83 formation of elongated/distorted micelles or vesicles [as 84 illustrated in Figure 1c] that cannot be captured using 85 currently available models. Such complex effects arise from 86 the heterogeneity in the solid surface chemistry and disordered 87 morphology/topology of the host confining material.

88 From a theoretical viewpoint, statistical physics is a powerful 89 framework to predict the complex behavior of surfactants in 90 bulk solution (including anomalous temperature effects on self91 assembly for instance). In particular, extended lattice gas 92 theory for monomers including a supralattice for the formation 93 of micelles was shown to capture most of the physical 94 phenomena involved in the phase diagram of these complex 95 objects. $^{30}$ This method was extended later by Bock and 96 Gubbins $^{31}$ to account for surface adsorption through the use of 97 surface interaction terms in the lattice gas Hamiltonian. ${ }^{30}$ 98 From a thermodynamic viewpoint, several models such as 99 those described hereafter have been proposed to describe 100 surfactant adsorption on solid surfaces. Empirical models have 101 been proposed to describe in an effective fashion the S-shaped 102 adsorption isotherms. This is the essence of the Sips model, ${ }^{32}$ 103 which corresponds to the Langmuir model with the pressure 104 raised to an empirical power $\alpha$. The Toth model falls in the 105 same category, as it consists of accounting empirically for 106 surface heterogeneity through a stretched Langmuir adsorption 107 isotherm. ${ }^{33}$ Other empirical approaches in this field consist of 108 combining different physical models such as Henry, Langmuir, 109 and BET adsorption isotherms to account for nonconventional 110 surfactant adsorption isotherms. ${ }^{34}$ More physical pictures have 111 been proposed such as the model by $\mathrm{Zhu}$ and $\mathrm{Gu}$, in which 112 adsorption is described as a two-step process with two 113 underlying equilibrium conditions (single-monomer adsorp114 tion and monomer recombination to form self-assemblies). ${ }^{35,36}$ 115 Other physical models such as those proposed by Temkin ${ }^{37}$ and Reed-Ehrlich ${ }^{38}$ rely on the quasi-chemical approximation 116 to account for lateral interactions within the adsorbed layer. 117 This approximation is an extension of the Bragg-Williams 118 approximation, in which the Langmuir model is augmented by 119 including a mean-field description of the lateral interactions 120 between adsorbed molecules. ${ }^{39}$

In spite of their physical basis, the models described above 122 do not provide a general formalism for surfactant adsorption, 123 as they address different aspects (lateral interaction or self- 124 assembly). In other words, a reliable thermodynamic 125 description of the behavior of surfactants at surfactant/surface 126 interfaces should include both lateral interactions and 127 transitions from adsorbed monomers to self-assembled objects. 128 This task is complex but also crucially needed, as the specific 129 adsorption type observed experimentally depends on many 130 parameters (surfactant type, surface chemistry, presence of 131 electrolytes/other fluid components, etc.). Here, we intend to 132 fill this gap by providing a generic theoretical picture of 133 surfactant adsorption through a phenomenological model 134 based on simple thermodynamic ingredients. In more detail, 135 this physical model is obtained by including physical 136 cooperative effects that account for both lateral interactions 137 between adsorbed molecules and self-assembly at the solid 138 surface through a site occupancy parameter that can be larger 139 than unity. It is important to recognize here that simply 140 accounting for lateral interactions in adsorption models is not 141 sufficient to describe the adsorption of mesoscopic objects at 142 the solid surface (even if such lateral interactions are physically 143 needed to account for self-assembly, a site occupancy $>1$ must 144 be considered to describe the adsorption of supramolecular 145 structures).

146

In practice, this model is derived by writing a constitutive 147 kinetic equation involving adsorption/desorption rates that are 148 dependent on the surfactant surface concentration to account 149 for facilitated or hindered adsorption. Using experimental data 150 for two surfactants on two mineral surfaces, this simple yet 151 realistic model is shown to capture different surfactant 152 adsorption types as observed upon varying the surfactant 153 affinity toward the surface. We note that the present model can 154 be extended to almost any surfactant adsorption/porous 155 surface type, as it has its roots in generic thermodynamic 156 concepts (fluid/surface affinity, occupancy/packing, fluid/fluid 157 interactions, etc.). The cooperative effects invoked in our 158 model to capture the complex adsorption phenomena 159 occurring at the surfactant/surface interface are believed to 160 be physically relevant as they can be rationalized using a simple 161 quasi-chemical adsorption model (which can be seen as a 162 Langmuir model in which interactions between adjacent 163 
164 adsorbed molecules are treated in a mean-field approximation). 165 It is also shown that the adsorption kinetics is drastically 166 affected by such cooperative effects, therefore offering an 167 additional mean to understand the physics of surfactant 168 adsorption (driving forces corresponding to lateral interactions 169 and surface self-assembly).

\section{THERMODYNAMIC MODEL}

170 To derive our model, let us consider the situation depicted in 171 Figure 2 with a solid surface made up of adsorption sites (a)

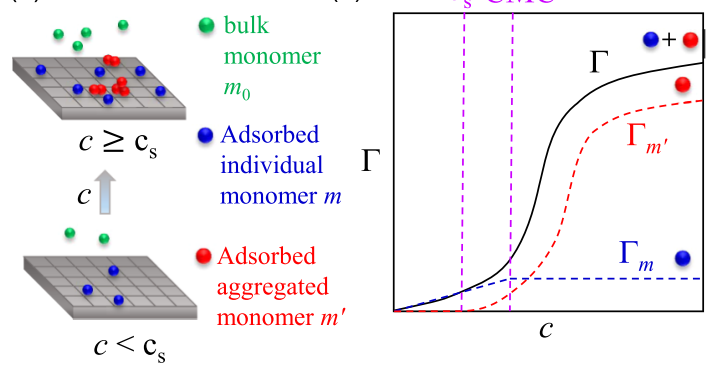

Figure 2. (a) Thermodynamic model of surfactant adsorption onto a solid surface in which the surface concentration $\Gamma$ as a function of the bulk concentration $c$ is the sum of a contribution $\Gamma_{\mathrm{m}}$ corresponding to adsorbed individual monomers $m$ and a contribution $\Gamma_{\mathrm{m}^{\prime}}$ corresponding to aggregated monomers $\mathrm{m}^{\prime}$. (b) Adsorption isotherm corresponding to the model shown in (a). The black line is the total surface concentration $\Gamma$, while the blue and red lines correspond to $\Gamma_{\mathrm{m}}$ and $\Gamma_{\mathrm{m}^{\prime}}$, respectively. For bulk concentration $c$ smaller than the critical surface concentration $c_{s}$, only isolated monomers $m$ get adsorbed at the surface-here following a Henry adsorption isotherm $\Gamma_{\mathrm{m}} \sim c$ but any other adsorption regime can be considered. For $c>$ $\mathrm{cmc}, \Gamma_{\mathrm{m}}$ plateaus as the bulk concentration of isolated monomers remains constant. For $c_{\mathrm{s}}<c<\mathrm{cmc}$, both isolated monomers $m$ and monomers in aggregated objects $m^{\prime}$ adsorb at the surface.

172 denoted as $s$. Each site $s$ can adsorb a single monomer $m$ (blue 173 sphere) or $n=1 / \beta$ aggregated monomers $m^{\prime}$ (red sphere), 174 where $n$ can be seen as the packing efficiency of aggregated 175 monomers. $\beta \in[0,1]$ is a key ingredient which renders our 176 model versatile, as it allows describing very different physical 177 situations. $\beta=1 / n$ describes the physical situation previously 178 considered by $\mathrm{Zhu}$ and $\mathrm{Gu},{ }^{35}$ where a single monomer is used 179 to aggregate with $n-1$ other monomers to form a column on 180 a single solid site $s$. In contrast, $\beta \rightarrow 1$ corresponds to very 181 weakly aggregated object, where each monomer $m^{\prime}$ lies at a 182 solid site $s$. Therefore, as will be illustrated in the present 183 paper, defining $\beta$ as a variable allows reproducing-at least in 184 an effective fashion-almost any aggregation type without 185 having to assume a given shape (micelle, hemi-micelle, vesicle, 186 disordered aggregate, etc.). In particular, the combined use of a 187 packing efficiency and surface concentration adsorption 188 constants allows describing both the adsorption of aggregated 189 monomers forming at the pore surface (below and above $\mathrm{cmc}$ ) 190 and the direct adsorption of micelles formed in the bulk 191 solution (above $\mathrm{cmc}$ ).

192 Having introduced the fundamental ingredients of our 193 model, its constitutive equations for adsorption equilibrium 194 and kinetics can be derived by writing simple mass-balance 195 equations between the free monomers $m_{0}$ (green spheres) in 196 solution $c=c\left[m_{0}\right]$, the solid surface sites $s$, the adsorbed 197 individual monomers $m$, and the adsorbed aggregated 198 monomers $\mathrm{m}^{\prime}$. Although such conditions can be written formally, rendering our model tractable requires an additional 199 assumption as follows. We introduce a surface critical 200 concentration $c_{\mathrm{s}}$ below which only individual monomers $m 201$ adsorb-this critical concentration can be seen as a minimum 202 concentration to observe the formation of aggregated (self- 203 assembled) structures at the solid surface. In this range, $c<c_{s}$, 204 surface phase equilibrium can be expressed as $s+m_{0} \rightleftharpoons m$ with 205 the underlying first-order kinetic equilibrium given by

206

$$
\frac{\partial \Gamma_{\mathrm{m}}(c, t)}{\partial t}=k_{\mathrm{A}} c\left[\Gamma^{\infty}-\Gamma_{\mathrm{m}}(c, t)\right]-k_{\mathrm{D}} \Gamma_{\mathrm{m}}(c, t)
$$

where $\Gamma_{\mathrm{m}}(c, t)$ is the surface concentration of individual 208 adsorbed monomers $m$ and $\Gamma^{\infty}$ is the surface site concentration 209 in which individual monomers $m$ can adsorb. The first and 210 second terms in the right hand side of eq 1 account for the 211 adsorption/desorption contributions over a time $\partial t\left(k_{\mathrm{A}}\right.$ and $k_{\mathrm{D}} 212$ are the adsorption and desorption rates, respectively). The 213 solution to this well-known mass-balance condition corre- 214 sponds to the Langmuir kinetics (with $k=k_{\mathrm{A}} / k_{\mathrm{D}}$ ) 215

$$
\Gamma_{\mathrm{m}}(c, t)=\frac{\Gamma^{\infty} c k}{1+c k}\left[1-\mathrm{e}^{-(1+k) k_{\mathrm{D}} t}\right]
$$

which converges in the stationary regime $(t \rightarrow \infty)$ toward the 217 Langmuir model $\Gamma_{\mathrm{m}}(c, \infty)=\Gamma^{\infty} c k /(1+c k)$. In passing, we 218 note that taking the limit $\Gamma^{\infty} \gg \Gamma_{\mathrm{m}}(c, \infty)$ allows recovering 219 the Henry regime as usually observed at very low 220 concentrations $c: \Gamma_{\mathrm{m}}(c, t)=\Gamma^{\infty} c k\left[1-\mathrm{e}^{-k_{\mathrm{D}} t}\right]$ with the long- 221 time limit $\Gamma_{\mathrm{m}}(c, \infty)=\Gamma^{\infty} c k$.

For $c \geq c_{s}$, both the individual monomers $m$ and aggregated 223 monomers $m^{\prime}$ adsorb in the surface sites $s$. As already 224 mentioned, using the concept of aggregated monomers, we 225 encompass into the same contribution, both the adsorption 226 and recombination of adsorbed surfactants into mesoscopic 227 assemblies (for $c>c_{\mathrm{s}}$ ) and the direct adsorption of micelles 228 formed in the bulk onto the solid surface (for $c>\mathrm{cmc}>c_{\mathrm{s}}$ ). 229 This is a specificity of our model in which the use of surface 230 concentration-dependent adsorption/desorption rates allows 231 treating in an effective yet physical fashion these complex 232 adsorption phenomena. Here, as a simplification that allows 233 straightforward comparison with experimental data without 234 changing fundamentally the physical basis of our model, we 235 assume that the adsorption of individual monomers $m$ occurs 236 on a much shorter timescale than the adsorption of aggregated 237 monomers $\mathrm{m}^{\prime}$. This implies that in the following kinetic 238 equation, $\Gamma_{\mathrm{m}}(c, t) \sim \Gamma_{\mathrm{m}}(c, \infty) \forall t$. Moreover, each surface site 239 is assumed to adsorb $n=1 / \beta$ aggregated monomers where the 240 packing efficiency $n$ allows accounting for nearly any self- 241 assembled object. With these approximations, surface phase 242 equilibrium for $c \geq c_{s}$ can be expressed as $s^{*}+m_{0} \rightleftharpoons m^{\prime}$ where 243 $*$ in $s^{*}$ indicates that only the surface sites that remain available 244 for aggregated monomers $m^{\prime}$ are considered. The correspond- 245 ing first-order kinetic equation for the adsorption/desorption 246 of the aggregated monomers $\mathrm{m}^{\prime}$ in such a process can be 247 expressed as

$$
\begin{aligned}
\frac{\partial \Gamma_{\mathrm{m}^{\prime}}(c, t)}{\partial t}= & k_{\mathrm{A}}^{\prime}\left(\Gamma_{\mathrm{m}^{\prime}}\right) c \times\left[\Gamma^{\infty}-\Gamma_{\mathrm{m}}(c, \infty)-\beta \Gamma_{\mathrm{m}^{\prime}}(c, t)\right] \\
& -k_{\mathrm{D}}^{\prime}\left(\Gamma_{\mathrm{m}^{\prime}}\right) \Gamma_{\mathrm{m}^{\prime}}(c, t)
\end{aligned}
$$

where $\Gamma_{\mathrm{m}^{\prime}}(c, t)$ is the surface concentration in aggregated 250 monomers $m^{\prime}$, while $\beta$ accounts for the fact that the adsorption 251 of a single monomer in aggregated objects only occupies a 252 fraction $\beta$ of the surface site (therefore, with these definitions, 253 
$254 \beta \Gamma_{\mathrm{m}^{\prime}}(c, t)$ is the number of such mesoscopic, i.e., aggregated, 255 objects). Moreover, to account for lateral interactions between 256 monomers in aggregated objects, we make the adsorption and 257 desorption rates $k_{\mathrm{A}}^{\prime}$ and $k_{\mathrm{D}}^{\prime}$ in the abovementioned equation 258 explicitly dependent on the surface concentration $\Gamma_{\mathrm{m}^{\prime}}$. At 259 equilibrium (i.e., in the stationary regime $\partial \Gamma_{\mathrm{m}^{\prime}} / \partial t=0$ ), for a 260 bulk concentration $c$, this kinetic equation leads to the 261 following solution

$$
\Gamma_{\mathrm{m}^{\prime}}(c, \infty)=\left[\Gamma^{\infty}-\Gamma_{\mathrm{m}}(c, \infty)\right] \times k^{\prime}\left(\Gamma_{\mathrm{m}^{\prime}}\right) c /\left[1+\beta c k^{\prime}\left(\Gamma_{\mathrm{m}^{\prime}}\right)\right]
$$

262

263 where $k^{\prime}\left(\Gamma_{\mathrm{m}^{\prime}}\right)=k_{\mathrm{A}^{\prime}}\left(\Gamma_{\mathrm{m}^{\prime}}\right) / k_{\mathrm{D}}^{\prime}\left(\Gamma_{\mathrm{m}^{\prime}}\right)$.

264 In summary, this model allows introducing a required degree 265 of complexity through collective effects in surfactant 266 adsorption that manifest themselves into two factors. First, 267 although isolated monomers are assumed to adsorb 268 independently of each other, lateral interactions between 269 monomers adsorbing into self-assemblies must be included. 270 Second, the formation of either ordered (e.g., hemimicelle and 271 vesicle) or distorted (e.g., elongated micelle) mesoscopic 272 assemblies is included in an effective fashion through the use of 273 a packing efficiency $n$. This generic model relies on a limited 274 yet important set of assumptions, namely, superimposition of 275 isolated and aggregated monomer adsorption, fast-isolated 276 monomer adsorption, and description of self-assemblies 277 through an effective parameter $n$. However, despite these 278 assumptions, as illustrated in the remaining of this paper, this 279 model allows deriving fundamental insights into the 280 thermodynamics and kinetics of surfactant adsorption from 281 simple experimental data. In particular, as shown below, a 282 merit of this model is that the adsorption-dependent dynamical 283 coefficients governing the adsorption kinetics of isolated and 284 aggregated monomers can be estimated from static adsorption 285 data (because the adsorption/desorption rates $k_{\mathrm{A}}^{\prime}$ and $k_{\mathrm{D}}^{\prime}$ 286 depend explicitly only on $\left.\Gamma_{\mathrm{m}^{\prime}}\right)$. Moreover, this versatile 287 model can be applied with almost no restriction regarding 288 the type of surfactants, surfaces, self-assemblies, thermody289 namic conditions, and so forth.

\section{RESULTS AND DISCUSSION}

290 To test our model, we consider two sets of experimental data, 291 which are representatives of different surfactant adsorption 292 behaviors. In more detail, we use the data by Denoyel and 293 Rouquerol who considered the adsorption at room temper294 ature of two polar (nonionic) surfactants onto silica-based 295 surfaces (TX100 onto silica and TX165 onto kaolinite clay). ${ }^{21}$ 296 As shown in Figure 3, for both systems, the adsorption 297 isotherms exhibit two regimes, which correspond to monomer 298 adsorption at low concentrations $c$ followed by a rapid increase 299 in the surface concentration, corresponding to surface self300 assembly at concentrations around the cmc. However, a major 301 difference between the two datasets lies in the monomer 302 adsorption regime in the low concentration range with a 303 slow-Henry-like-regime for Figure $3 \mathrm{a}$ and a rapid304 Langmuir-like-regime for Figure 3b (we note that in general, 305 a Langmuir model can be assumed by default, as the Henry law 306 is simply its asymptotic limit). Because the two surfactants 307 considered here are similar nonpolar molecules with an $\mathrm{OH}$ 308 group at their end, the origin of this difference has to be found 309 in the surface chemistry of the different surfaces. Typically, for 310 the kaolin sample, as discussed by Denoyel and Rouquerol, ${ }^{21}$ 311 the observed strong adsorption phenomenon is thought to 312 occur on a basal plane-more exactly, the basal plane made up
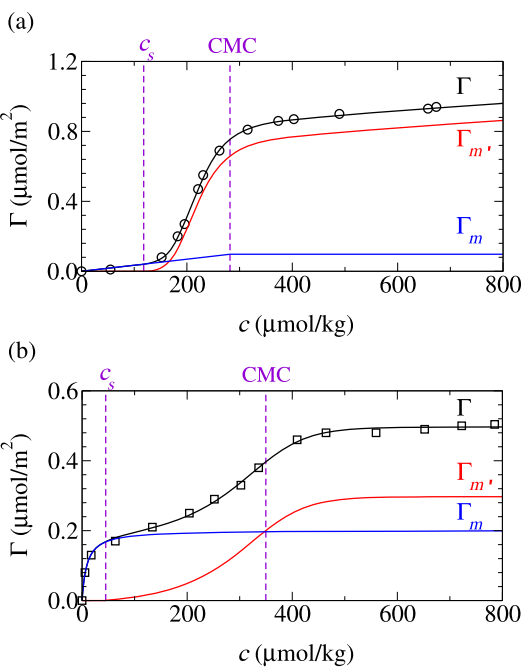

Figure 3. Surfactant adsorption isotherms at $T=298 \mathrm{~K}$ showing the surface concentration of surfactants $\Gamma$ onto a silica-based surface as a function of the bulk concentration $c$ : (a) TX100 surfactant on quartz silica and (b) TX165 surfactant on kaolin. The black symbols are the experimental data taken from Denoyel and Rouquerol ${ }^{21}$ with the black line corresponding to smoothed interpolation data. For both systems, the blue and red lines show the predictions of our model for the adsorption of isolated and aggregated monomers, respectively (by construction, the sum of these two contributions is equal to the experimental data). A Henry law and a Langmuir law were used to describe the isolated monomer adsorption in $(\mathrm{a}, \mathrm{b})$, respectively. The vertical dashed lines indicate the critical surface concentration $c_{\mathrm{s}}$ and cmc.

of aluminol sites, as the surface concentrations were found to 313 be $\mathrm{pH}$-independent (adsorption sites on this basal plane do not 314 form amphoteric charges with $\mathrm{pH}) .^{21}$ In contrast, because the 315 weak adsorption phenomenon displayed in Figure 3 for silica is 316 found to be $\mathrm{pH}$-sensitive, it is assumed to occur on neutral 317 adsorption sites that become charged upon increasing the $\mathrm{pH}$. 318

We use the following procedure to apply our model to the 319 experimental data $\Gamma^{\exp }(c)$ shown in Figure 3. First, to define 320 the surface critical concentration $c_{s}$, the interpolated exper- 321 imental data are fitted against a Langmuir adsorption isotherm 322 over a concentration range $\left[0, c_{\max }\right]$. Although the fit is 323 accurate for small $c_{\max }$, the fit does not provide satisfactory 324 results for large $c_{\max }$ (because a simple Langmuir or Henry 325 adsorption isotherm cannot describe the raw experimental 326 adsorption data over a large-concentration range). At this 327 stage, it is decided to define $c_{\mathrm{s}}$ as the maximum value $c_{\max }$, for 328 which a correlation coefficient of $R^{2}=0.99$ is obtained. 329 Although the specific cutoff value used is arbitrary, it should be 330 emphasized here that slightly different values would lead to 331 very similar predictions (in practice, with variations in the 332 degree of agreement with experimental data that falls within 333 the experimental error bar). This allows us to have a good 334 approximation to describe the monomer surface concentration 335 $\Gamma_{\mathrm{m}}(c, \infty)$ for $c<c_{\mathrm{s}}$. Here, it is worth mentioning that fitting the 336 Henry or Langmuir model to low-concentration data requires 337 that enough experimental data points are available to ensure 338 rigorous fitting and, hence, robust physical parameterization. 339 As an illustration, Figure S2 shows the different adsorption 340 isotherms obtained as $c_{\mathrm{s}}$ is varied from $c_{\mathrm{s}} \sim 0$ to $c_{\mathrm{s}}>\mathrm{cmc}$. 341 Second, having a fitted model for $\Gamma_{\mathrm{m}}(c, \infty)$, one can estimate 342 the contribution corresponding to the adsorbed aggregated 343 monomers $\Gamma_{\mathrm{m}^{\prime}}(c, \infty)$ by subtracting $\Gamma_{\mathrm{m}}(c, \infty)$ from $\Gamma^{\exp }(c), 344$ 
345 that is, $\Gamma_{\mathrm{m}^{\prime}}(c, \infty)=\Gamma^{\exp }(c)-\Gamma_{\mathrm{m}}(c, \infty)$. Third, $k^{\prime}\left(\Gamma_{\mathrm{m}^{\prime}}\right)$ can be 346 readily estimated from $\Gamma_{\mathrm{m}^{\prime}}(c, \infty)$ by inverting eq 4

$$
k^{\prime}\left(\Gamma_{\mathrm{m}^{\prime}}\right)=\frac{\Gamma_{\mathrm{m}^{\prime}}(c, \infty)}{c\left[c-\Gamma_{\mathrm{m}}(c, \infty)-\beta \Gamma_{\mathrm{m}^{\prime}}(c, \infty)\right]}
$$

348 As shown in Figure 3, for both systems, the model including 349 cooperative effects applies accurately to the experimental data. 350 From a practical viewpoint, as described above, applying our 351 model to available experiments requires to determine the 352 following parameters. Although the Langmuir (Henry) 353 constant $k_{\mathrm{H}}\left(k_{\mathrm{L}}\right)$ must be fitted together with $c_{\mathrm{s}}$ from the 354 low-concentration range, the maximum adsorbed amount $\Gamma^{\infty}$ 355 is determined from the high-concentration range.

356 Figure 4 shows $k^{\prime}\left(\Gamma_{\mathrm{m}^{\prime}}\right)$ as a function of $\Gamma_{\mathrm{m}^{\prime}}$ for the two 357 systems considered here (TX100 on quartz silica and TX165

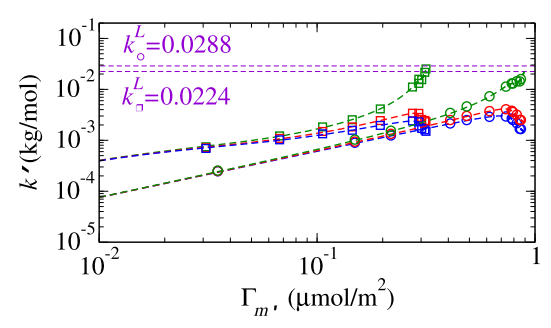

Figure 4. Adsorption constant $k^{\prime}\left(\Gamma_{\mathrm{m}^{\prime}}\right)$ for the aggregated monomers $m^{\prime}$ as a function of their surface concentration $\Gamma_{m^{\prime}}$, as extracted from the experimental adsorption data shown in Figure 3. The circles are for TX100 adsorption on quartz silica, while the squares are for TX165 on kaolin (experimental data taken from ref 21). For each system, the blue, red, and green data denote data obtained for $\beta=0.2$, $\beta=0.5$, and $\beta=1.0$, respectively. The two horizontal dashed lines in purple indicate the Langmuir adsorption constants that best match the experimental adsorption isotherms in the high-concentration range $c>c_{s}$.

358 on kaolin). It should be noted that the model applied to the 359 isothermal adsorption data does not allow estimating $\beta$. This is 360 a drawback of our model, but $\beta$ can be estimated 361 independently of $k^{\prime}\left(\Gamma_{\mathrm{m}^{\prime}}\right)$ from adsorption kinetic data, as 362 shown in the last part of this paper. Figure 4 shows that $363 k^{\prime}\left(\Gamma_{\mathrm{m}^{\prime}}\right)$ increases with increasing $\Gamma_{\mathrm{m}^{\prime}}$ for a given $\beta$, therefore 364 pointing to the existence of collective, that is, cooperative, 365 effects in surfactant adsorption (otherwise $k^{\prime}\left(\Gamma_{\mathrm{m}^{\prime}}\right)$ would 366 remain constant). Physically, this behavior indicates that 367 cooperative effects lead to enhanced adsorption with already368 adsorbed molecules, facilitating adsorption of additional 369 monomers either in the same adsorption sites (when $\beta \neq 1$ ) 370 and/or in neighboring adsorption sites $(\beta=1)$. As expected 371 from eq 5 , upon decreasing $\beta$, the fraction of available sites to 372 adsorb aggregated monomers increases, so that $k^{\prime}\left(\Gamma_{\mathrm{m}^{\prime}}\right)$ 373 decreases. This is due to the fact that $k^{\prime}\left(\Gamma_{\mathrm{m}^{\prime}}\right)$ is an effective 374 adsorption constant, so that low $\beta$ corresponds to systems that 375 tend to self-assemble easily (therefore not requiring large 376 adsorption constants to pack efficiently at the solid surface). 377 Figure 4 also shows for the two systems considered here the 378 Langmuir adsorption constant $k^{\mathrm{L}}=k_{\mathrm{A}} / k_{\mathrm{D}}$, which was estimated 379 by fitting the concentration range beyond the critical surface 380 concentration $c>c_{s}$. In more detail, by restricting the fitting 381 procedure to the region where the surface concentration 382 increases rapidly with concentration, it is possible to describe 383 semiquantitatively the data using a simple Langmuir model 384 with shifted concentrations $\tilde{c} \sim c-c_{s}$. As shown in Figure 4, 385 except for large surface concentrations $\Gamma_{\mathrm{m}^{\prime}}$, regardless of the system considered, the Langmuir constant $k^{\mathrm{L}}$ overestimates the 386 adsorption constant $k^{\prime}\left(\Gamma_{\mathrm{m}^{\prime}}\right)$ predicted using our model (Figure 387 S3 in the Supporting Information shows the fits of the high- 388 concentration experimental data to the Langmuir model). This 389 result is due to the fact that the Langmuir model does not 390 describe cooperative adsorption, so a larger effective constant 391 is needed to capture the increasing adsorption rate upon 392 increasing the surface concentration.

393

For a given surfactant/surface couple, the parameters 394 involved in the present model are derived from available 395 experimental data. To assess the physical validity and 396 robustness of our model, it is important to connect its 397 underlying parameters to existing theoretical frameworks. In 398 this context, as stated in the Introduction, statistical physics 399 offers an efficient and accurate formalism to describe 400 cooperative adsorption effects. In particular, the quasi-chemical 401 approximation $^{39}$ allows deriving a simple expression for the 402 surface concentration that accounts for lateral interactions 403 between adsorbed monomers. Here, for the sake of brevity, we 404 provide the main thermodynamic ingredients of this important 405 model, which goes well beyond the Langmuir model (an exact 406 derivation can be found in the Supporting Information). Let us 407 consider free monomers in a bulk solution that can adsorb 408 onto surface sites. Assuming additive lateral interactions 409 between an adsorbed molecule and its neighbors, the 410 adsorption energy $E$ of a monomer cluster made of $x 411$ monomers is $E \sim x w$, where $w$ is the interaction with a single 412 neighbor. Phase equilibrium between the adsorbed monomers 413 and the free monomers in solution at a temperature $T$ implies 414 that the chemical potential is equal in the two phases, that is, $\mu 415$ $=\mu_{\mathrm{b}}$ (the subscript $\mathrm{b}$ refers to the bulk solution). In the quasi- 416 chemical approximation, as shown in the Supporting 417 Information, the chemical potential of the adsorbed phase 418 writes $\mu=\mu_{0}+k_{\mathrm{B}} T \ln [\theta /(1-\theta)]+z_{0} k_{\mathrm{B}} T / 2 \ln [(\gamma-1+419$ $2 \theta)(1-\theta) / \theta(\gamma+1-2 \theta)]$, where $\theta \in[0,1]$ is the site 420 average occupancy, $z_{0}$ is the number of neighboring sites $\left(z_{0}=421\right.$ 4 for a surface), and $\gamma=[1-4 \theta(1-\theta)(1-\eta)]^{1 / 2}$ (with $\eta=422$ $\left.\exp \left[-w / k_{\mathrm{B}} T\right]\right)$. In this expression, the reference chemical 423 potential $\mu_{0}=\left[z_{0} w / 2+\epsilon_{0}\right] / k_{\mathrm{B}} T$ corresponds to the energy of 424 an adsorbed monomer at full saturation (which includes an 425 energy contribution with the surface $\sim \epsilon_{0}$ and an energy 426 contribution with all neighboring adsorbed monomers $\sim z_{0} w / 427$ $2)$. By noting that $\theta=\beta \Gamma_{\mathrm{m}^{\prime}} /\left(\Gamma^{\infty}-\Gamma_{\mathrm{m}}\right)$ and $1-\theta=\left(\Gamma^{\infty}-\Gamma_{\mathrm{m}} 428\right.$ $\left.-\beta \Gamma_{\mathrm{m}^{\prime}}\right) /\left(\Gamma^{\infty}-\Gamma_{\mathrm{m}}\right)$, the previous expression leads to the 429 following expression for $\Delta \mu=\mu-\mu_{0}$ 430

$$
\begin{aligned}
\frac{\Delta \mu}{k_{\mathrm{B}} T}= & \ln \left[\frac{\beta \Gamma_{\mathrm{m}^{\prime}}}{\Gamma^{\infty}-\Gamma_{\mathrm{m}}-\beta \Gamma_{\mathrm{m}^{\prime}}}\right]+\frac{z_{0}}{2} \ln \left[\frac{\Gamma^{\infty}-\Gamma_{\mathrm{m}}-\beta \Gamma_{\mathrm{m}^{\prime}}}{\beta \Gamma_{\mathrm{m}^{\prime}}}\right] \\
& +\frac{z_{0}}{2} \ln \left[\frac{(\gamma-1)\left(\Gamma^{\infty}-\Gamma_{\mathrm{m}}\right)+2 \beta \Gamma_{\mathrm{m}^{\prime}}}{(\gamma+1)\left(\Gamma^{\infty}-\Gamma_{\mathrm{m}}\right)-2 \beta \Gamma_{\mathrm{m}^{\prime}}}\right]
\end{aligned}
$$

Taking the bulk concentration $c=c_{0}$ as the concentration at 432 the reference point $\mu_{0}$ and assuming that the concentration 433 remains low enough, we can write $\Delta \mu$ for the bulk phase as 434 $\Delta \mu_{\mathrm{b}}=k_{\mathrm{B}} T \ln c / c_{0}$. By inverting eq 5 , we obtain $c=1 / k^{\prime}\left(\Gamma_{\mathrm{m}^{\prime}}\right) \times 435$ $\Gamma_{\mathrm{m}^{\prime}} /\left[\Gamma^{\infty}-\Gamma_{\mathrm{m}}-\beta \Gamma_{\mathrm{m}^{\prime}}\right]$, which leads to the following 436 expression upon insertion in $\Delta \mu_{\mathrm{b}}$

437

$$
\frac{\Delta \mu_{\mathrm{b}}}{k_{\mathrm{B}} T}=\ln \left[\frac{\beta \Gamma_{\mathrm{m}^{\prime}}}{\Gamma^{\infty}-\Gamma_{\mathrm{m}}-\beta \Gamma_{\mathrm{m}^{\prime}}}\right]-\ln \left[\frac{c_{0} \times k^{\prime}\left(\Gamma_{\mathrm{m}^{\prime}}\right)}{\beta}\right]
$$

To verify that our model of cooperative adsorption is 439 consistent with a description of interacting adsorbed species, 440 
441 we can check that the chemical potential equality as defined in 442 the quasi-chemical approximation is obeyed. To do so, by 443 noting that the first term on the right-hand side, that is, $\mu^{*}=$ $444 k_{\mathrm{B}} T \ln \left[\beta \Gamma_{\mathrm{m}^{\prime}} /\left(\Gamma^{\infty}-\Gamma_{\mathrm{m}}-\beta \Gamma_{\mathrm{m}^{\prime}}\right)\right]$, is identical in eqs 6 and 7 , 445 we can compare $\Delta \mu-\mu^{*}$ and $\Delta \mu_{\mathrm{b}}-\mu^{*}$. As shown in Figure 4465 , when using the values for $k^{\prime}$ in Figure 4, a good agreement is

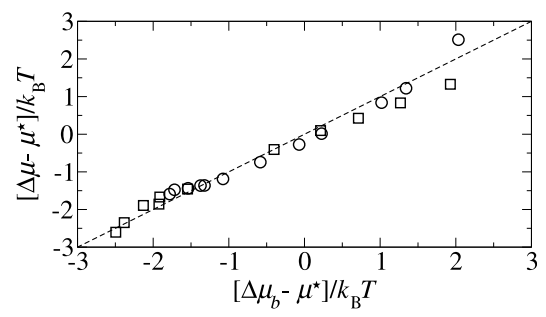

Figure 5. Comparison between the chemical potential shift for bulk and adsorbed surfactants, as predicted within the quasi-chemical approximation using the data derived from our model. The circles and squares refer to the data for TX100 on quartz silica and TX165 on kaolin, respectively (experimental data taken from ref 21). The dashed line is a guide to the eye, which indicates chemical potential equality between the bulk and adsorbed phases.

447 obtained between the two chemical potentials for both TX100 448 on quartz silica and TX165 on kaolin. When establishing such 449 a comparison, $\eta=\exp \left[-w / k_{\mathrm{B}} T\right]$ and $c_{0}$ were used as adjustable 450 parameters, but we note that they are the only fitting 451 variables-we found that $\eta \sim 3.3$ for TX100 on silica and $\eta$ $452 \sim 4.0$ for TX165 on kaolinite. More importantly, it was verified 453 a posteriori that these values for $\eta$, which lead to $w \sim-1.2 k_{\mathrm{B}} T$ 454 for TX100 on silica and $w \sim-1.4 k_{\mathrm{B}} T$ for TX165 on kaolinite, 455 are physically relevant, as discussed in what follows. First, as 456 expected for cooperative effects leading to facilitated 457 adsorption, $w$ is negative, so that it corresponds to attractive 458 lateral interactions between adsorbed neighbors. Second, $w$ is 459 of the order of $k_{\mathrm{B}} T$, as required to observe cooperative 460 adsorption (indeed, for lower, i.e., less, negative lateral 461 interactions, thermal motion and therefore desorption would 462 prevail). Third, the stronger attractive interaction $w$ for TX165 463 is consistent with the fact that this molecule is similar to 464 TX100 but with a longer alkyl chain-16 versus 9-10 carbon 465 groups. In practice, the two datasets used are for different 466 surfaces- which could affect the comparison made here-but 467 we recall that $\eta$ is related to the interaction between two 468 adsorbed molecules and can, therefore, be considered to be 469 mostly dependent on their molecular nature/chemistry. A few 470 remarks are in order here. First, although our thermodynamic 471 model is found to be consistent with the quasi-chemical 472 approximation, the latter is insufficient to fully describe 473 surfactant adsorption over the whole concentration range. 474 Indeed, the quasi-chemical approximation is only relevant in 475 the concentration range $c>c_{s}$, where the surfactant aggregates 476 at the solid surface to form mesoscopic structures (which stem 477 from lateral interactions/collective effects). In contrast, in the 478 low-concentration range, such collective effects are assumed to 479 be absent, so the quasi-chemical approximation is not relevant. 480 Second, even if $w$ should formally depend on the surfactant 481 type only, it is expected to be a function of the solid surface 482 nature in practice. Indeed, considering that the quasi-chemical 483 approximation is a mean-field treatment, effective parameters 484 such as $w$ are expected to depend-even if weakly-on the 485 strength/nature of the surfactant/solid interactions (in other 486 words, the lateral interactions in adsorbed surfactants are necessarily mediated to some extent by the solid/surfactant 487 interactions).

As will be shown here, the present model has strong 489 implications in terms of surfactant adsorption/desorption 490 kinetics on surfaces. In particular, changes in the adsorption/ 491 desorption rates induce drastic variations in the characteristic 492 time corresponding to the transient regime leading to 493 thermodynamic equilibrium. Although this feature is not 494 specific to our model (because underlying kinetics in the 495 Langmuir and Henry models also depend on the adsorption/ 496 desorption constant rates), the introduced concept of $\Gamma_{\mathrm{m}^{\prime}} 497$ dependence of $k_{\mathrm{A}}$ and $k_{\mathrm{D}}$ leads to rich and complex kinetics. In 498 this respect, it should be emphasized that only such a level of 499 complexity allows capturing the intriguing adsorption kinetics 500 observed experimentally for surfactant adsorption. In partic- 501 ular, all typical non-Langmuirian adsorption dynamics 502 observed in transient adsorption experiments but also in 503 breakthrough curves, which resist available modeling frame- 504 works, point to the existence of cooperative adsorption effects 505 and, more generally, complex collective phenomena. ${ }^{16}$ To 506 illustrate the influence of cooperative effects on adsorption 507 kinetics, the dynamical equation given in eq 3 was solved 508 numerically for different bulk concentrations $c$. For TX100 509 adsorption on silica, this leads to the time evolution shown in 510 Figure 6 (the same data for TX165 on kaolin are not shown $511 \mathrm{f} 6$ here for the sake of clarity but can be found in Figure S1 in the 512 Supporting Information). Many choices can be made for 513 $k_{\mathrm{A}}^{\prime}\left(\Gamma_{\mathrm{m}^{\prime}}\right)$ and $k_{\mathrm{D}}^{\prime}\left(\Gamma_{\mathrm{m}^{\prime}}\right)$ because static adsorption data only 514 provide information on $k^{\prime}\left(\Gamma_{\mathrm{m}^{\prime}}\right)=k_{\mathrm{A}}^{\prime}\left(\Gamma_{\mathrm{m}^{\prime}}\right) / k_{\mathrm{D}}^{\prime}\left(\Gamma_{\mathrm{m}^{\prime}}\right)$. Two 515

(a)

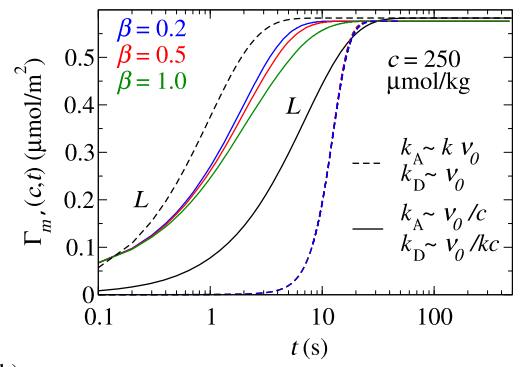

(b)

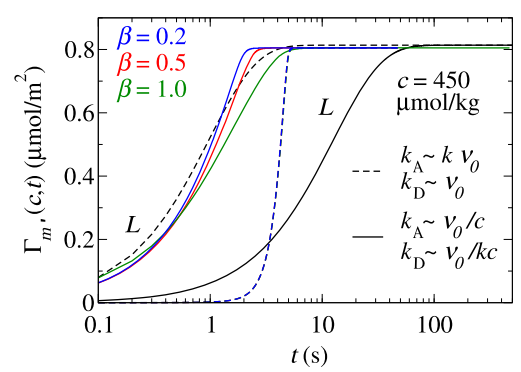

Figure 6. Adsorption kinetics as determined by solving numerically eq 3 , showing $\Gamma_{\mathrm{m}^{\prime}}$ as a function of time $t$ for TX100 on silica for two bulk concentrations: (a) $c=250 \mu \mathrm{mol} / \mathrm{kg}$ and (b) $c=450 \mu \mathrm{mol} / \mathrm{kg}$. The color lines denote the data obtained using the cooperative model with $\beta=0.2$ (blue), $\beta=0.5$ (red), and $\beta=1.0$ (green), while the black lines correspond to kinetics predicted using the Langmuir kinetic model with an adsorption/desorption constant $k^{\mathrm{L}}$ that best matches the experimental adsorption isotherm (see text). In each case, the dashed lines correspond to the case $k_{\mathrm{A}} \sim \nu_{0} k$ and $k_{\mathrm{D}} \sim \nu_{0}$, while the solid lines correspond to $k_{\mathrm{A}} \sim \nu_{0} / c$ and $k_{\mathrm{D}} \sim \nu_{0} / k c$. Note that our model predicts that the color dashed lines (i.e., $k_{\mathrm{A}} \sim k$ and $k_{\mathrm{D}} \sim$ constant) are superimposed. 
516 illustrative situations were considered as shown in Figure 6: $517(1) k_{\mathrm{A}}^{\prime} \sim \nu_{0} k^{\prime}\left(\Gamma_{\mathrm{m}^{\prime}}\right)$ and $k_{\mathrm{D}}^{\prime} \sim \nu_{0}$ and $(2) k_{\mathrm{A}}^{\prime} \sim \nu_{0} / c$ and $k_{\mathrm{D}}^{\prime} \sim \nu_{0} /$ $518 k^{\prime}\left(\Gamma_{\mathrm{m}^{\prime}}\right) \mathcal{c}$, where $\nu_{0}$ is a characteristic constant in $s^{-1}$ that sets 519 the typical timescale (because it is used as a constant 520 throughout this study, it does not affect the discussion 521 provided below). We emphasize that the two cases considered 522 here are asymptotic limits as both $k_{\mathrm{A}}^{\prime}$ and $k_{\mathrm{D}}^{\prime}$ should depend on $523 k^{\prime}$ and hence $\Gamma_{\mathrm{m}^{\prime}}$ in general. For each situation, we also 524 consider the effect of the aggregation parameter $\beta$, which is 525 varied between $\beta=0.2$ and $1(n=1 / \beta$ is the number of 526 aggregated monomers that can be packed into a single 527 adsorption site). Figure 6 also shows for each situation the 528 kinetics obtained using the Langmuir kinetic model with (1) $k_{\mathrm{A}}^{\mathrm{L}}$ $529 \sim \nu_{0} k^{\mathrm{L}}$ and $k_{\mathrm{D}}^{\mathrm{L}} \sim \nu_{0}$ and (2) $k_{\mathrm{A}}^{\mathrm{L}} \sim \nu_{0} / c$ and $k_{\mathrm{D}}^{\mathrm{L}} \sim \nu_{0} / k^{\mathrm{L}} c$, where $530 k^{\mathrm{L}}$ is the Langmuir adsorption constant that best matches the 531 experimental adsorption isotherm in the high-concentration 532 range $c>c_{\text {s }}$ (see Figure 4 and its caption).

533 Examining in detail the two situations considered here 534 allows gaining insights into the role of cooperative effects on 535 surfactant adsorption/desorption kinetics in porous media. In 536 particular, this illustrates how application of the present model 537 to experimental adsorption kinetic data could be used to probe 538 adsorption and desorption properties including the aggregation 539 constant $\beta$.

540

541

542

543

544

545

546

547

548

549

550

551

552

553

554

555

556

557

558

559

560

561

562

563

564

565

566

567

568

569

570

571

572

573
- Case $\left[k_{\mathrm{A}}^{\prime} \sim k^{\prime}\right.$, while $k_{\mathrm{D}}^{\prime} \sim$ constant]. With these assumptions, inserting the expression for $k^{\prime}$ given in eq 5 into the kinetics described by eq 3 shows that the adsorption rate $k_{\mathrm{A}}^{\prime}$ is constant (i.e., independent of $\beta$ ). Therefore, in this case, both $k_{\mathrm{A}}^{\prime}$ and $k_{\mathrm{D}}^{\prime}$ are constant, so that the adsorption kinetics is independent of the aggregation parameter $\beta$ for all concentrations $c$ (see color dashed lines in Figure 6). Moreover, comparison with the ideal Langmuir model for this case indicates that our cooperative model predicts a much slower kinetics as $k^{\prime} \lesssim k^{\mathrm{L}}$ for all $c$ (as shown in Figure 4). Indeed, at a constant desorption rate, the kinetics becomes faster with increasing adsorption rate.

- Case $\left[k_{\mathrm{A}}^{\prime} \sim\right.$ constant, while $\left.k_{\mathrm{D}}^{\prime} \sim 1 / k^{\prime}\right]$. With these assumptions, $\beta$ significantly affects the observed adsorption kinetics. As can be inferred from eq 5, $k^{\prime}$ increases with $\beta$, so that $k_{\mathrm{D}}^{\prime} \sim 1 / k^{\prime}$ decreases. As can be directly illustrated using a simple Langmuir kinetic equation, the adsorption kinetics becomes slower with decreasing desorption constant $k_{\mathrm{D}}$ while maintaining $k_{\mathrm{A}}$ constant. This interpretation is consistent with the data shown in Figure 6 for $k_{\mathrm{D}}^{\prime} \sim 1 / k^{\prime}$ and $k_{\mathrm{A}}$ constant, where it is observed that the adsorption kinetics becomes slower with increasing $\beta$. Finally, for a given concentration with $k_{\mathrm{D}} \sim 1 / k^{\prime}$ and $k_{\mathrm{A}}$ constant, we observe that the simple Langmuir kinetics is significantly slower than that observed with cooperative effects. This result is consistent with our previous explanation on the role of the desorption rate at constant $k_{\mathrm{A}}$ because $k^{\mathrm{L}}>k^{\prime}$ for all $c$ leads to $k_{\mathrm{D}}^{\mathrm{L}}<k_{\mathrm{D}}^{\prime}$ and therefore a slower kinetics for the Langmuir model. Further study is in progress to fully explore the impact of such cooperative effects on adsorption kinetics using available experimental kinetic data.

\section{CONCLUSIONS}

574 In conclusion, we developed a simple physical model of the 575 thermodynamics and kinetics of surfactant adsorption onto surfaces that accounts for cooperative effects inherent to such 576 complex objects. By cooperative effects, we refer here to strong 577 lateral interactions between adsorbed surfactants but also 578 intramolecular and intermolecular interactions responsible for 579 their propensity to form mesoscopic (supramolecular) 580 structures. With this model, important collective driving forces 581 that cannot be ignored for such self-assembling molecules are 582 taken into account to describe the nonconventional static and 583 dynamic adsorption behavior observed experimentally when 584 surfactant solutions are set in contact with solid surfaces. Such 585 collective effects are also at the root of surface aggregation at 586 solution/air interfaces, which stems from mutual interactions 587 between adsorbed molecules. ${ }^{40}$ This cooperative behavior was 588 also recently discussed in the context of surfactant adsorption 589 at the water/oil interface, where adsorbed oil molecules were 590 found to enhance surfactant adsorption. ${ }^{41}$ In practice, the 591 simple thermodynamic model derived in this paper involves a 592 simple kinetic formalism involving adsorption/desorption rates 593 that vary with the surfactant surface concentration. Such a 594 formalism can be extended to any class of objects that is 595 expected to involve adsorption cooperative effects such as ionic 596 liquids, ${ }^{42}$ long-chain molecules (e.g., normal alkanes), ${ }^{43}$ and so 597 forth. Moreover, although all cases treated here involved a 598 surface concentration $c_{\mathrm{s}}$ lower than the $\mathrm{cmc}$, our model also 599 deals without any further development to nonwetting 600 situations where surface aggregation occurs beyond its bulk 601 counterpart. The fact that, in all cases, the adsorption isotherm 602 reaches a plateau at a concentration close to the $\mathrm{cmc}$ can be 603 rationalized as follows. The $\mathrm{cmc}$ corresponds to the chemical 604 potential at which the surfactants in bulk solution condense to 605 form dense surfactant objects (micelles). Within perturbation 606 theory, near a solid surface, the chemical potential where such 607 condensation occurs can be written as the bulk value shifted by 608 a surface interaction contribution.

609

Once applied to available experimental data, this framework 610 provides a valuable tool to infer key quantities that govern the 611 microscopic behavior of any adsorbed surfactant onto various 612 solid surfaces including surface self-assembly into ordered or 613 disordered structures. More generally, this robust and versatile 614 model, which is found to be consistent with rigorous 615 microscopic treatments such as the quasi-chemical approx- 616 imation in statistical physics of surface adsorption, can be 617 extended in principle to surfactant adsorption but also 618 transport in porous materials. Beyond immediate practical 619 implications, the results reported here about the nonstandard 620 surfactant adsorption thermodynamics and kinetics in porous 621 materials also raise new challenging questions. In particular, 622 owing to cooperative effects in surfactant adsorption, strong 623 departure from the adsorption/dynamics interplay observed 624 for more classical fluids is to be expected in agreement with 625 experimental observations in breakthrough or injection experi- 626 ments. This issue is particularly important, as diffusion is 627 usually considered as the main transport mechanism leading to 628 adsorption on surfaces. The present work offers a well- 629 grounded thermodynamic basis to address such questions. $\quad 630$

\section{ASSOCIATED CONTENT}

The Supporting Information is available free of charge at 633 https://pubs.acs.org/doi/10.1021/acs.jpcb.0c08226. 


\section{AUTHOR INFORMATION}

\section{Corresponding Authors}

640 Daniela Bauer - IFP Energies Nouvelles, 92852 Rueil

641 Malmaison, France; Email: daniela.bauer@ifpen.fr

642 Benoit Coasne - Université Grenoble Alpes, CNRS, LIPhy,

64338000 Grenoble, France; 이잉.org/0000-0002-3933-

644 9744; Email: benoit.coasne@univ-grenoble-alpes.fr

\section{Authors}

646 Zaineb Zaafouri - IFP Energies Nouvelles, 92852 Rueil

647 Malmaison, France; Université Grenoble Alpes, CNRS,

648 LIPhy, 38000 Grenoble, France

649 Guillaume Batôt - IFP Energies Nouvelles, 92852 Rueil

650 Malmaison, France

651 Carlos Nieto-Draghi - IFP Energies Nouvelles, 92852 Rueil

652 Malmaison, France; 이이이.org/0000-0001-5956-9259

653 Complete contact information is available at:

654 https://pubs.acs.org/10.1021/acs.jpcb.0c08226

655 Notes

656 The authors declare no competing financial interest.

\section{ACKNOWLEDGMENTS}

658 The authors are grateful to Elise Lorenceau and Julie Wolanin 659 from Laboratoire Interdisciplinaire de Physique for helpful 660 comments. Z.Z. acknowledges Ph.D. funding at Univ. 661 Grenoble Alpes from IFP Energies Nouvelles.

\section{$662 \square$ REFERENCES}

663 (1) Rosen, M. J.; Kunjappu, J. T. Surfactants and Interfacial 664 Phenomena; John Wiley \& Sons Inc.: Hoboken, New Jersey, 2012.

665 (2) Raffa, P.; Wever, D. A. Z.; Picchioni, F.; Broekhuis, A. A. 666 Polymeric Surfactants: Synthesis, Properties, and Links to Applica667 tions. Chem. Rev. 2015, 115, 8504-8563.

668 (3) Ruiz-Ángel, M. J.; Carda-Broch, S.; Torres-Lapasió, J. R.; García669 Álvarez-Coque, M. C. Retention Mechanisms in Micellar Liquid 670 Chromatography. J. Chromatogr. A 2009, 1216, 1798-1814.

671 (4) Olkowska, E.; Polkowska, Z.; Namieśnik, J. Analytics of 672 Surfactants in the Environment: Problems and Challenges. Chem. 673 Rev. 2011, 111, 5667-5700.

674 (5) Bera, A.; Kumar, T.; Ojha, K.; Mandal, A. Adsorption of 675 Surfactants on Sand Surface in Enhanced Oil Recovery: Isotherms, 676 Kinetics and Thermodynamic Studies. Appl. Surf. Sci. 2013, 284, 8767799.

678 (6) Kathel, P.; Mohanty, K. K. Wettability Alteration in a Tight Oil 679 Reservoir. Energy Fuels 2013, 27, 6460-6468.

680 (7) Zhou, Y.; Wu, X.; Zhong, X.; Sun, W.; Pu, H.; Zhao, J. X. 681 Surfactant-Augmented Functional Silica Nanoparticle Based Nano682 fluid for Enhanced Oil Recovery at High Temperature and Salinity. 683 ACS Appl. Mater. Interfaces 2019, 11, 45763-45775.

684 (8) Israelachvili, J. N. Intermolecular and Surface Forces; Academic 685 Press: London, San Diego, 1991.

686 (9) Soni, S. S.; Sastry, N. V.; Joshi, J. V.; Seth, E.; Goyal, P. S. Study 687 on the Effects of Nonelectrolyte Additives on the Phase, 688 Thermodynamics, and Structural Changes in Micelles of Silicone 689 Surfactants in Aqueous Solutions From Surface Activity, Small Angle 690 Neutron Scattering, and Viscosity Measurements. Langmuir 2003, 19, $6916668-6677$.

692 (10) Strey, R. I. Experimental Facts: Water-Nonionic Surfactant 693 Systems, and the Effect of Additives. Ber. Bunsen Ges. Phys. Chem. 694 1996, 100, 182-189.
(11) Corti, M.; Degiorgio, V.; Zulauf, M. Nonuniversal Critical 695 Behavior of Micellar Solutions. Phys. Rev. Lett. 1982, 48, 1617.

(12) Wilcoxon, J. P.; Martin, J. E.; Odinek, J. Anomalous Phase 697 Separation Kinetics Observed in a Micelle Solution. Phys. Rev. Lett. 698 1995, 75, 1558.

699

(13) Atkin, R.; Craig, V. S. J.; Biggs, S. Adsorption Kinetics and 700 Structural Arrangements of Cationic Surfactants on Silica Surfaces. 701 Langmuir 2000, 16, 9374-9380.

702

(14) Király, Z.; Findenegg, G. H. Calorimetric Study of the 703 Adsorption of Short-Chain Nonionic Surfactants on Silica Glass and 704 Graphite: Dimethyldecylamine Oxide and Octyl Monoglucoside. 705 Langmuir 2000, 16, 8842-8849.

706

(15) Lugo, D.; Oberdisse, J.; Karg, M.; Schweins, R.; Findenegg, G. 707 H. Surface Aggregate Structure of Nonionic Surfactants on Silica 708 Nanoparticles. Soft Matter 2009, 5, 2928-2936. 709

(16) Kwok, W.; Nasr-El-Din, H. A.; Hayes, R. E.; Sethi, D. Static and 710 Dynamic Adsorption of a Non-Ionic Surfactant on Berea Sandstone. 711 Colloids Surf., A 1993, 78, 193-209.

(17) Denoyel, R.; Giordano, F.; Rouquerol, J. Thermodynamic 713 Study of Non-Ionic-Anionic Surfactant Mixtures: Micellization and 714 Adsorption on Silica. Colloids Surf., A 1993, 76, 141-148. 715

(18) Zhang, R.; Somasundaran, P. Advances in Adsorption of 716 Surfactants and their Mixtures at Solid/Solution Interfaces. Adv. 717 Colloid Interface Sci. 2006, 123-126, 213-229. , Special Issue in 718 Honor of Dr. K. L. Mittal

(19) Penfold, J.; Thomas, R. K. Probing Surfactant Adsorption at the 720 Solid-Solution Interface by Neutron Reflectometry. Interface Sci. 721 Technol. 2007, 14, 87-115.

(20) Levitz, P.; Van Damme, H. Fluorescence Decay Study of the 723 Adsorption of Nonionic Surfactants at the Solid-Liquid Interface. 2. 724 Influence of Polar Chain Length. J. Phys. Chem. 1986, 90, 1302-1310. 725 (21) Denoyel, R.; Rouquerol, J. Thermodynamic (Including 726 Microcalorimetry) Study of the Adsorption of Nonionic and Anionic 727 Surfactants onto Silica, Kaolin, and Alumina. J. Colloid Interface Sci. 728 1991, 143, 555-572.

(22) Penfold, J.; Staples, E.; Tucker, I.; Cummins, P. Adsorption of 730 Nonionic Surfactants on Silica Sol Particles: The Effects of Sol Type 731 and Concentration, Surfactant Type, Concentration, and Temper- 732 ature. J. Phys. Chem. 1996, 100, 18133-18137.

(23) Qiao, Y.; Schönhoff, M.; Findenegg, G. H. 2H NMR 734 Investigation of the Structure and Dynamics of the Nonionic 735 Surfactant C12E5 Confined in Controlled Pore Glass. Langmuir 736 2003, 19, 6160-6167.

(24) Shin, T. G.; Müter, D.; Meissner, J.; Paris, O.; Findenegg, G. H. 738 Structural Characterization of Surfactant Aggregates Adsorbed in 739 Cylindrical Silica Nanopores. Langmuir 2011, 27, 5252-5263. 740

(25) Levitz, P. E. Adsorption of Non Ionic Surfactants at the Solid/ 741 Water Interface. Colloids Surf., A 2002, 205, 31-38.

(26) Findenegg, G. H.; Eltekov, A. Y. Adsorption Isotherms of 743 Nonionic Surfactants in SBA-15 Measured by Micro-Column 744 Chromatography. J. Chromatogr. A 2007, 1150, 236-240. 745

(27) Esumi, K.; Ueno, M. Structure-Performance Relationships in 746 Surfactants; Taylor \& Francis Inc.: Bosa Roca, United States, 2003; 747 Vol. 112.

(28) Oberdisse, J. Small Angle Neutron Scattering and Model 749 Predictions for Micelle-Decorated Colloidal Silica Beads. Phys. Chem. 750 Chem. Phys. 2004, 6, 1557-1561.

(29) Lugo, D. M.; Oberdisse, J.; Lapp, A.; Findenegg, G. H. Effect of 752 Nanoparticle Size on the Morphology of Adsorbed Surfactant Layers. 753 J. Phys. Chem. B 2010, 114, 4183-4191.

(30) Vause, C. A.; Walker, J. S. Effects of Orientational Degrees of 755 Freedom in Closed-Loop Solubility Phase Diagrams. Phys. Lett. A 756 1982, 90, 419-424.

(31) Bock, H.; Gubbins, K. Anomalous Temperature Dependence of 758 Surfactant Self-Assembly From Aqueous Solution. Phys. Rev. Lett. 759 2004, 92, 135701.

760

(32) Sips, R. On the Structure of a Catalyst Surface. II. J. Chem. Phys. 761 1950, 18, 1024-1026. 
763 (33) Toth, J. State Equation of the Solid-Gas Interface Layers. Acta 764 Chim. Hung. 1971, 69, 311-328.

765 (34) Redlich, O.; Peterson, D. L. A Useful Adsorption Isotherm. J. 766 Phys. Chem. 1959, 63, 1024.

767 (35) Zhu, B.-Y.; Gu, T. General Isotherm Equation for Adsorption 768 of Surfactants at Solid/Liquid Interfaces. Part 1. Theoretical. J. Chem. 769 Soc., Faraday Trans. 1 1989, 85, 3813-3817.

770 (36) Zhu, B.-Y.; Gu, T. Surfactant Adsorption at Solid-Liquid 771 Interfaces. Adv. Colloid Interface Sci. 1991, 37, 1-32.

772 (37) Temkin, M. I. Adsorption Equilibrium and Kinetics of Process 773 on Non-Homogeneous Surfaces and in the Interaction between 774 Adsorbed Molecules. Zh. Fiz. Chim. 1941, 15, 296-332.

775 (38) Reed, D. A.; Ehrlich, G. Surface Diffusion, Atomic Jump Rates 776 and Thermodynamics. Surf. Sci. 1981, 102, 588-609.

777 (39) Hill, T. L. Statistical Mechanics: Principles and Selected 778 Applications; Dover Publications: New York, 1987.

779 (40) Fainerman, V. B.; Miller, R. Surface Tension Isotherms for 780 Surfactant Adsorption Layers Including Surface Aggregation. 781 Langmuir 1996, 12, 6011-6014.

782 (41) Fainerman, V. B.; Aksenenko, E. V.; Kovalchuk, V. I.; Mucic, 783 N.; Javadi, A.; Liggieri, L.; Ravera, F.; Loglio, G.; Makievski, A. V.; 784 Schneck, E.; et al. New View of the Adsorption of Surfactants at the 785 Water/Alkane Interface - Competitive and Cooperative Effects of 786 Surfactant and Alkane Molecules. Adv. Colloid Interface Sci. 2020, 279, 787102143 .

788 (42) Ori, G.; Villemot, F.; Viau, L.; Vioux, A.; Coasne, B. Ionic 789 Liquid Confined in Silica Nanopores: Molecular Dynamics in the 790 Isobaric-Isothermal Ensemble. Mol. Phys. 2014, 112, 1350-1361.

791 (43) Falk, K.; Pellenq, R.; Ulm, F. J.; Coasne, B. Effect of Chain 792 Length and Pore Accessibility on Alkane Adsorption in Kerogen. 793 Energy Fuels 2015, 29, 7889-7896. 